\title{
Tumours composed of fat are no longer a simple diagnosis: an overview of fatty tumours with a spindle cell component
}

\author{
Aoife J McCarthy, Runjan Chetty
}

Department of Pathology, Laboratory Medicine Program, University Health Network, University of Toronto, Toronto, Canada

\section{Correspondence to} Professor Runjan Chetty, Department of Pathology, Laboratory Medicine Program, University Health Network Toronto General Hospital, Toronto, Ontario M5G 2C4, Canada; runjan.chetty@gmail. com

Received 20 December 2017 Accepted 24 December 2017 Published Online First 22 January 2018
Check for updates

To cite: McCarthy AJ, Chetty R. J Clin Pathol 2018;71:483-492.

\begin{abstract}
This is a review of the morphological spectrum of fatty tumours containing a component of spindle cells, highlighting the immunohistochemical and cytogenetic workup that is now mandatory for accurate diagnosis, with the goal of providing a practical approach for practising surgical pathologists. There have been significant advances in recent years in classifying and understanding the pathogenesis of fatty tumours with spindle cells, based on the correlation of histological, immunohistochemical and cytogenetic/molecular findings. In spite of this, morphological diagnosis and accurate classification of fatty tumours with spindle cells can be challenging to diagnostic pathologists. A group of three lesions: spindle cell lipoma, mammarytype myofibroblastoma and cellular angiofibroma share morphological features and are united by retinoblastoma protein ( $p R b)$ loss. Closely allied to these lesions, especially spindle cell lipoma is the newly designated atypical spindle cell lipomatous tumour, which shares morphological, immunohistochemical and cytogenetic features with the trio of tumours lacking nuclear pRb. All of these lesions lack MDM2 and CDK4 amplification as well and separation is based on clinical features, principally location. Atypical lipomatous tumour or well-differentiated liposarcoma shows retention of $\mathrm{pRb}$ but overexpression and amplification of MDM2. Fatty tumours with spindle cells need to be extensively sampled, with careful attention paid to cellular atypia and location, and they need to have immunohistochemical workup with pRb, MDM2, desmin, CD34 and p16. In addition, cytogenetic analysis for MDM2 and CDK4 amplification has become crucial for the proper identification of these lesions.
\end{abstract}

\section{INTRODUCTION}

Soft tissue sarcomas are diverse mesenchymal malignancies that account for approximately $1 \%$ of adult solid tumours. ${ }^{1}$ Despite their rarity, they hold a diagnostic and intellectual mystique that transcends their relative infrequency under a general diagnostic pathologist's microscope. Adipocytic tumours represent the most common type of mesenchymal tumours. ${ }^{2}$ Awareness and classification of atypical and malignant adipocytic tumours have evolved rapidly in the last few years due to a better biological understanding from large series, and with new entities being described by virtue of the application of ancillary techniques such as cytogenetics, molecular genetics and immunohistochemistry. With this has come a level of morphological complexity that necessitates a more than desultory examination of fatty tumours microscopically.

The morphological, and often the immunohistochemical, similarities between fatty tumours can cause diagnostic challenges for even experienced surgical pathologists. We present a synthesis of recently described fatty lesions with a spindle cell component, the differential diagnosis and a review of the currently known morphological, immunohistochemical and molecular features of these tumours. The dilemma confronting the non-expert pathologist dealing with a fatty tumour with spindle cells is nomenclature. Calling a lesion a liposarcoma triggers a particular management algorithm together with labelling the patient as having a sarcoma. Failing to recognise a lesion as potentially locally recurring is the other side of the diagnostic quandary. Thus, the goal of this overview is to highlight key histological features, provide diagnostic pointers to help categorise these lesions as accurately as possible and separate them from other key histological mimics enabling pathologists to make informed and appropriate comments regarding behaviour and management. It is not meant to be a comprehensive expose on each entity, many of which are dealt with in specialised soft tissue textbooks, but a practical perspective when confronted by lesions composed of fat and spindle cells (table 1).

\section{Spindle cell lipoma}

Nomenclature

Spindle cell and pleomorphic lipomas (SC/PL) are characterised by a morphological continuum and have identical cytogenetics, are considered a single entity. $^{3}$

\section{Clinical features}

They are relatively uncommon, comprising approximately $1.5 \%$ of adipocytic neoplasms and being 60 times less frequently encountered than simple lipomas. ${ }^{4}$ SC/PLs are solitary lesions that grow slowly in a very restricted anatomical distribution, namely, the posterior neck, shoulder and back. They occur most frequently (90\%) in adult males, in their $40 \mathrm{~s}-60 \mathrm{~s}$ and usually measure $3-5 \mathrm{~cm}$ in diameter. ${ }^{5}$ Thus, the clinical feature of note aiding the diagnosis of SC/PL is location.

\section{Microscopic findings}

SC/PLs are well-circumscribed, often encapsulated tumours, located in the dermis and subcutis, 


\begin{tabular}{|c|c|c|c|c|}
\hline Lesion & Clinical & Microscopy & $\mathrm{IHC}$ & Cytogenetics \\
\hline $\mathrm{SCL}$ & $\begin{array}{l}\text { Middle age males, posterior } \\
\text { back, neck, shoulders }\end{array}$ & $\begin{array}{l}\text { Bland to atypical, }+/- \text { lipoblasts, ropey collagen, } \\
\text { myxoid stroma }\end{array}$ & $\begin{array}{l}\text { CD34+; SMA+; pRb-; desmin- ; } \\
\text { MDM2-l+; } \\
\text { CDK4-; p16-l+ }\end{array}$ & $\begin{array}{l}\text { Deletion of } 13 q 14 ; \text { no } \\
\text { amplification of MDM2 or } \\
\text { CDK4 }\end{array}$ \\
\hline MTMF & Groin, trunk, lower extremities & $\begin{array}{l}\text { Well-circumscribed, bland, diffuse collagenous } \\
\text { stroma }\end{array}$ & $\begin{array}{l}\text { CD34+; desmin+; SMA+; EMA+; pRb-; } \\
\text { MDM2-; CDK4-; p16- }\end{array}$ & $\begin{array}{l}\text { Deletion of } 13 q 14 ; \text { no } \\
\text { amplification of } M D M 2 \text { or } \\
\text { CDK4 }\end{array}$ \\
\hline ASCLT & $\begin{array}{l}\text { Adult males, limbs and limb } \\
\text { girdles }\end{array}$ & $\begin{array}{l}\text { III-defined, variable: cellularity, atypia, fat cell size; } \\
\text { lipoblasts in } 50 \% \text {, multinucleated cells }\end{array}$ & $\begin{array}{l}\text { CD34+; desmin }+; \mathrm{S} 100+; \mathrm{pRb}+\text { or }- \text { in } \\
50 \% ; \\
\text { MDM2-; CDK4-; } 16-\end{array}$ & $\begin{array}{l}\text { Deletion of } 13 q 14 \text { in } 50 \%- \\
60 \% \text {; no amplification of } \\
\text { MDM2 or CDK4 }\end{array}$ \\
\hline ALT & $\begin{array}{l}\text { Anywhere deep-seated } \\
\text { (retroperitoneum, mediastinum) } \\
\text { except posterior back, neck, } \\
\text { shoulders }\end{array}$ & $\begin{array}{l}\text { Adipocyte size variation, low, cellularity, mild-to- } \\
\text { moderate atypical hyperchromatic spindle cells, } \\
\text { lipoblasts, rare mitoses, myxoid stroma }\end{array}$ & $\begin{array}{l}\text { CD34-; desmin-; pRb+; MDM2+; CDK4+; } \\
\text { p16+ }\end{array}$ & $\begin{array}{l}\text { Amplification of } M D M 2 \text { and } \\
\text { CDK4 }\end{array}$ \\
\hline
\end{tabular}

+ , positive; $-/+$, mainly negative, but can be positive; $+/-$, mainly positive, but can be negative.

ALT, atypical lipomatous tumour; ASCLT, atypical spindle cell lipomatous tumour; CA, cellular angiofibroma; DDL, dedifferentiated liposarcoma; EMA, epithelial membrane antigen;

ER, oestrogen receptor; IHC, immunohistochemistry; MTMF, mammary-type myofibroblastoma; PR, progesterone receptor; pRb, retinoblastoma protein; SCL, spindle cell lipoma;

SMA, smooth muscle actin.

composed of a mixture of mature adipocytes and spindle cells, frequently set in a myxoid matrix (figure 1A). The spindle cells are cytologically bland, arranged in short fascicles in a 'school of fish' pattern. ${ }^{6}$ Ropey stromal collagen (thick, refractile, eosinophilic collagen) is a characteristic feature (figure $1 \mathrm{~B}$ ). The ratio of the mature fat to the spindle cells can vary significantly. Infrequently, fat is present in a small amount in 'low-fat' and 'fat-free' spindle cell lipomas (SCLs), which can cause diagnostic challenges. ${ }^{5}$ Vessels may be prominent, and may be arborising and thick-walled. Occasionally, mast cells are numerous.

Atypical cells range from rare to frequent and are characterised by hyperchromatic, enlarged nuclei, located in both fatty

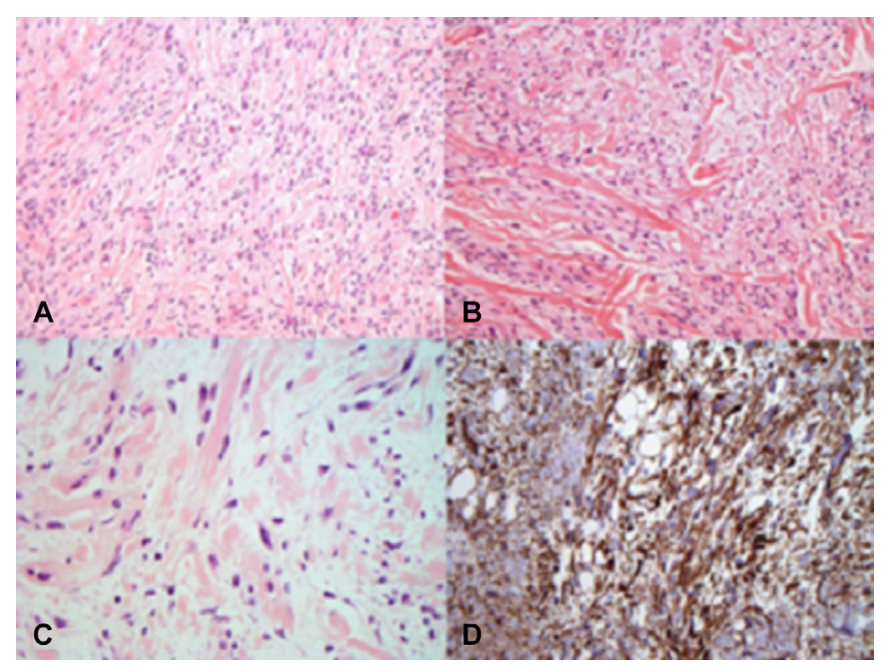

Figure 1 Spindle cell lipoma (SCL) consists of an admixture of fat and spindle cells which are usually uniform and bland (A). The stromal collagen is disposed as birefringent, so-called ropey appearing strands (B). The stroma can be myxoid and occasionally the spindle cells manifest mild atypia (C). SCL are strongly CD34 positive (D). and non-fatty stromal areas (figure 1C). These atypical cells may form a semi-circle of nuclei, designated as 'floret cells'.

It is important to note that lipoblasts, which are cells with hyperchromatic nuclei that are indented or sharply scalloped due to lipid-rich monovacuolated or multivacuolated cytoplasmic droplets, may be seen occasionally in otherwise morphologically and cytogenetically typical examples of SC/PL, particularly in pleomorphic lipomas. ${ }^{67}$

\section{Immunohistochemistry and cytogenetics}

The spindle cells are CD34 positive (figure 1D), while CD99 and bcl-2 are usually positive too. S100 stains fat cells only. Nuclear retinoblastoma protein $(\mathrm{pRb})$ is lost in SC/PL and typically, p16 immunoexpression is not seen in the adipocytes or the spindle-shaped tumour cell nuclei, although up to $20 \%$ of cases can also be positive. ${ }^{8}$ Both MDM2 and CDK4 are usually negative, but again a small proportion of cases can show immunopositivity.

The tumour suppressor gene, $R B 1$, encoding the $\mathrm{pRb}$ located at $13 \mathrm{q} 14$, is deleted in these lesions, ${ }^{9}$ explaining the loss of expression of immunohistochemical marker $\mathrm{pRb} .{ }^{8} \mathrm{SC} / \mathrm{PL}$ lacks amplification of MDM2 and CDK4.

\section{Treatment and prognosis}

Local excision is almost always curative and metastases have never been reported.

\section{Mammary-type myofibroblastoma \\ Clinical features}

Mammary-type myofibroblastoma (MTMF) is a benign mesenchymal lesion, first described in the breast, which is now known to have a wide anatomical distribution, most commonly occurring in the groin, inguinal area, trunk and lower extremity. ${ }^{1011}$ They are well-circumscribed lesions, with a median size of $5-7 \mathrm{~cm}$, occurring twice as commonly in males in their 50s.

The degree of morphological overlap between MTMF, SCL and cellular angiofibroma has raised the question whether these 


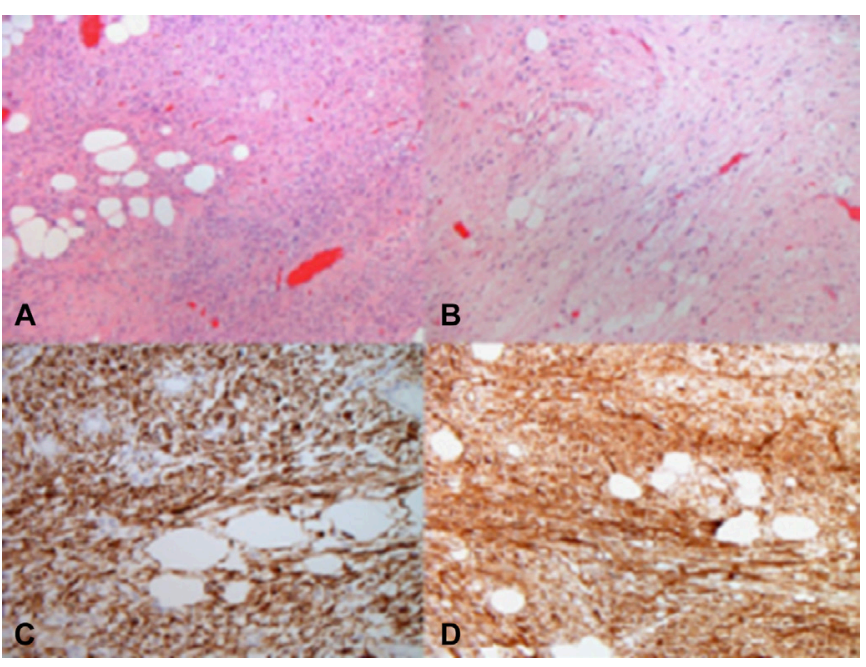

Figure 2 Mammary-type myofibroblastoma (MTMF) also is composed of variable proportions of fat and uniform spindle cells $(A)$ set within a stroma that can be myxoid (B). MTMF are desmin (C) and CD34 positive (D).

benign tumours are truly distinct entities or represent points along a single spectrum of genetically related (all have a deletion or rearrangement of $13 \mathrm{q} 14$ with subsequent loss of nuclear $\mathrm{pRb}$ ) tumours. ${ }^{10}$

\section{Microscopic findings}

MTMF is a well-circumscribed, bland, spindle cell proliferation, reminiscent of SCL, with a variably prominent lipomatous component (figure 2A). The tumour cells generally show no atypia, and the adipocytes show minimal variation in size and shape. The stroma is typically collagenous, and occasionally hyalinised or myxoid (figure 2B). Morphological heterogeneity has also been described including cellular, epithelioid and neurilemmoma-like variants. ${ }^{12-15}$

\section{Immunohistochemistry and cytogenetics}

Immunohistochemically, in most cases (up to 90\%), the lesional cells express desmin and CD34 diffusely (figure 2C,D). ${ }^{10}$ Approximately $40 \%$ also are smooth muscle actin (SMA) and epithelial membrane antigen positive. Very occasional cases are S100, MDM2 and CDK4 positive too. Most MTMFs show loss of $\mathrm{pRb}$ nuclear expression as a result of $13 \mathrm{q} 14$ abnormalities. ${ }^{16}$

\section{Differential diagnosis}

The differential diagnosis of MTMF can be broad and somewhat dependent on the anatomic location and/or the presence of unusual morphological features. ${ }^{10}$ However, in most situations the differential diagnosis is limited and most notably includes other tumours in the $13 \mathrm{q} / \mathrm{Rb}$ family, namely SC/PL and cellular angiofibroma. $^{16-19}$

1. SCL occurs almost exclusively in the posterior shoulder, neck or upper back region, has more consistently present ropey, refractile collagen bundles and, in contrast to MTMF, is negative for desmin while being positive for CD34. ${ }^{410}$

2. Cellular angiofibroma is generally limited to the inguinal or groin region and contains wispy rather than ropey or thick collagen, usually has a more limited adipocytic element and has a prominent component of rounded stromal vessels, often with perivascular fibrosis or hyalinization. ${ }^{18} 20$ See below for fuller discourse on cellular angiofibroma.
3. Myolipomas/lipoleiomyomas are also benign tumours and may be distinguished from MTMF by more conspicuous smooth muscle morphology with tighter fascicles. In addition, they do not share the cytogenetics of MTMF and do not show loss of $\mathrm{pRb}$.

Other entities with more clinical significance that are occasionally considered in the differential diagnosis of MTMF include atypical lipomatous tumour (ALT), desmoid tumour (fibromatosis) and solitary fibrous tumour (SFT). It is important to distinguish these from MTMF as these tumours have a significant risk for recurrence and, in rare cases (most notably in SFT), metastasis. ${ }^{102122}$

4. ALT: MTMF can be distinguished from ALT by the lack of significant nuclear atypia, the lack of variation in adipocyte size and the absence of lipoblasts. By immunohistochemistry, ALTs are positive for MDM2 and CDK4, reflecting amplification of $12 \mathrm{q} 13-15$, which can also be demonstrated using fluorescence in situ hybridisation (FISH). ${ }^{23}$ Nuclear $\mathrm{pRb}$ expression is retained in ALT, which can serve as another helpful marker in this distinction. ${ }^{16}$

5. Desmoid tumour/fibromatosis: unlike MTMF, desmoid tumours comprise long fascicles of spindle cells and frequently demonstrate nuclear expression of $\beta$-catenin. ${ }^{24}$ They are not well circumscribed and are desmin negative.

6. SFT may occasionally enter the differential diagnosis of MTMF, particularly the 'fat-forming' variant of SFT. These two entities share a CD34-positive immunophenotype, but SFTs have a patternless architecture and are not characterised by loss of $\mathrm{pRb}$ (immunohistochemically and cytogenetically), and do not express desmin. Instead, SFT harbours recurrent gene rearrangements resulting in overexpression of STAT6, a sensitive and specific marker for SFT. ${ }^{25-27}$

\section{Treatment and prognosis}

MTMFs do not recur, even if incompletely excised, and have no potential for metastasis. ${ }^{10}$

\section{Cellular angiofibroma Clinical features}

Cellular angiofibroma is an uncommon benign mesenchymal tumour, occurring mainly in the genital region of both females and males. ${ }^{18} 20$ Extragenital localisations have also been rarely reported, including in the retroperitoneum, pelvic and lumbar regions, anus, urethra, trunk and oral mucosa. 18 20 28-30

\section{Microscopic findings}

Cellular angiofibromas are typically well-circumscribed lesions, localised to the superficial soft tissue. They are composed of bland spindle-shaped (sometimes epithelioid) cells, arranged in no particular pattern, in an occasionally myxoid stroma with wispy collagen (figure 3A). Numerous small-sized to medium-sized, thick-walled blood vessels with mural hyalinization are typically present (figure 3B). ${ }^{1820}$ Vessels may contain fibrin thrombi, vessel wall inflammation and degenerative changes with hemosiderin. A mature adipocytic component is variably seen. ${ }^{18} 20$

\section{Immunohistochemistry and cytogenetics}

Cellular angiofibromas are usually positive for CD34 (30\%$60 \%$ ), progesterone receptor and oestrogen receptor (over 90\%) and negative for desmin. ${ }^{18}$ Loss of nuclear $\mathrm{pRb}$ staining is a characteristic finding. In keeping with this immunohistochemical pattern, essentially all cases show a loss of genetic material from 


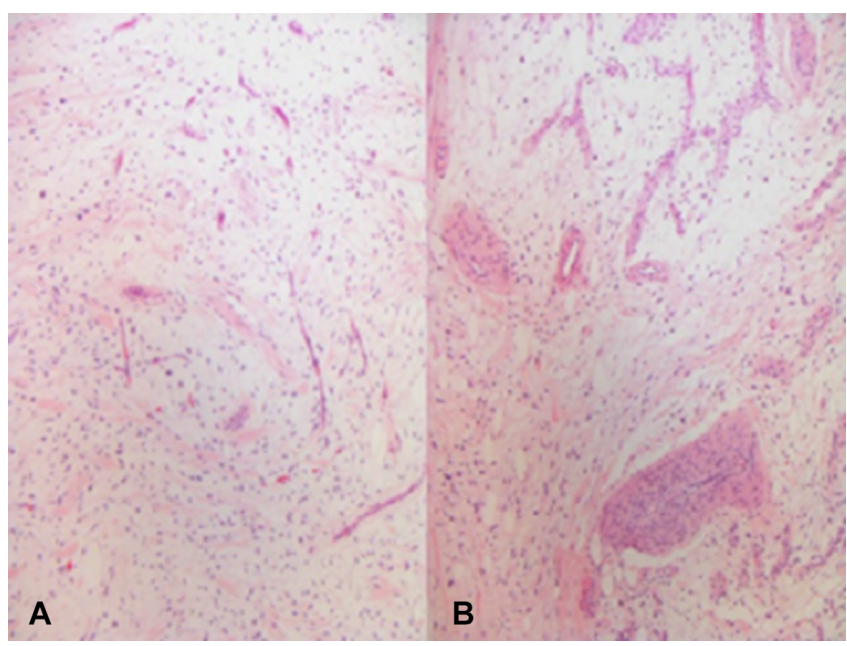

Figure 3 Cellular angiofibroma consists of uniform cells often set within a myxoid stroma with less prominent stromal collagen ('wispy') compared with spindle cell lipoma (A). Characteristically, but not exclusively, vessels of varying calibre are encountered (B).

the $13 \mathrm{q} 14$ region, as indicated by monoallelic deletion of $R B 1$ and FOXO1. ${ }^{19}$

\section{Differential diagnosis}

1. SFT: see 'differential diagnosis' subsection of the 'mammarytype myofibroblastoma' section above.

2. Angiomyofibroblastoma could potentially be confused with cellular angiofibroma, due to its localisation to the genital region. ${ }^{3132}$ Angiomyofibroblastomas are composed of more epithelioid, and often plasmacytoid, cells, arranged in cords and nests, preferentially located around blood vessels. The tumour cells express desmin but are only rarely positive for CD34. ${ }^{18}$ Nuclear pRb immunopositivity is usual.

3. Deep ('aggressive') angiomyxoma also involves the pelvic region and tends to be quite large, displaying an infiltrative growth pattern with entrapment of mucosal glands, fat, muscle and nerves. The prominent vascular component shows hyalinization. However, myoid bundles, mostly adjacent to medium-sized blood vessels, are a typical finding. ${ }^{18}$ There is variable positivity for desmin, smooth muscle actin and $\mathrm{CD} 34^{3132}$ and retention of nuclear $\mathrm{pRb}$.

\section{Prognosis and treatment}

There are a few reported cases in the literature of cellular angiofibroma with atypia or sarcomatous features. The sarcomatous component described shows variable features, including ALT, pleomorphic liposarcoma and pleomorphic sarcoma not otherwise specified. ${ }^{1820293334}$ Although the follow-up of all of these reported cases did not disclose any local recurrence or metastases, ${ }^{33}$ the biological significance of atypia and sarcomatous transformation in cellular angiofibromas remains uncertain.

\section{Atypical spindle cell lipomatous tumour Nomenclature}

This category of fatty tumours is a repository for tumours composed of atypical spindle cells and a variable fat component. Initially, cases were called ALTs or well-differentiated liposarcoma (WDL). However, it soon became apparent that a subset of spindle cell fatty tumours had distinct immunohistochemical and cytogenetic features and included entities such as spindle cell liposarcoma, atypical SCL and fibrosarcoma-like lipomatous

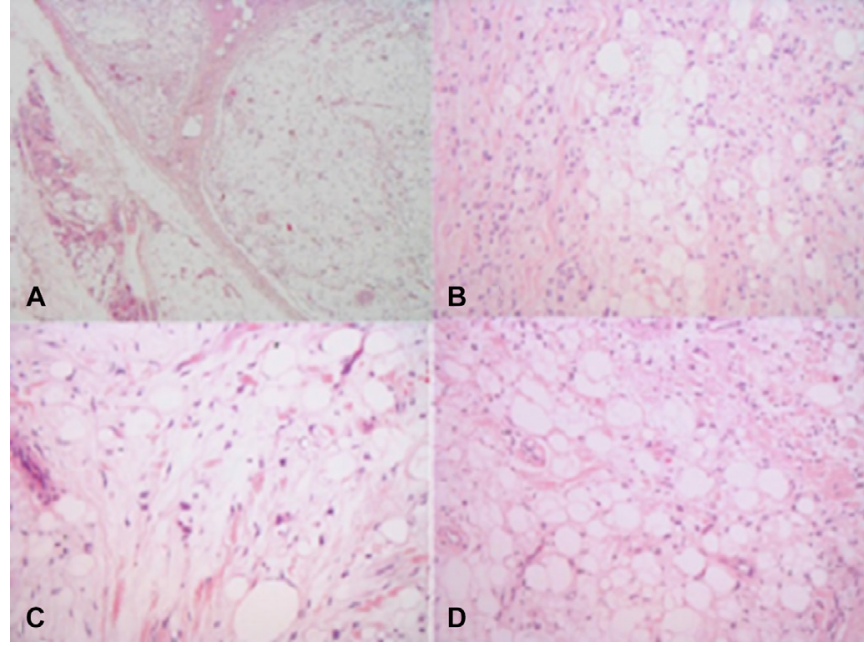

Figure 4 Atypical spindle cell lipomatous tumour has a lobular appearance with a fibrous pseudocapsule $(A)$ and is made up of uniform spindle cells, admixed with a variably prominent adipocytic component, set in a fibrous, collagenous or myxoid stroma (B). Spindle cells have ovoid nuclei with variably hyperchromatic chromatin and generally regular nuclear contours (C). The fatty component shows variation in fat cell size (D).

tumour. ${ }^{8}$ This entity should not be confused with ALT, which is also known as WDL.

\section{Clinical features}

Atypical spindle cell lipomatous tumour (ASCLT) constitutes a distinct category of adipocytic neoplasms of intermediate biological potential, which most frequently affects adult males in their $50 \mathrm{~s}$, has a median size of $5 \mathrm{~cm}$ and has a predilection for the limbs and limb girdles. ${ }^{35}$

These neoplasms were first described by Dei Tos et $a l^{36}$ in 1994, when they characterised a series of six cases that they designated 'spindle cell liposarcoma', which they considered to be variants of WDL.

\section{Microscopic findings}

Microscopically, ASCLTs have an ill-defined fibrous pseudo-capsule (figure 4A), and are composed of a vaguely lobular neoplastic proliferation of relatively uniform spindle cells, admixed with a variably prominent adipocytic component, set in a fibrous, collagenous or myxoid stroma (figure 4B). The proportions of spindle cells, adipocytes and extracellular matrix varies, resulting in a broad range of microscopic appearances. ${ }^{35}$

The neoplastic spindle cells are elongated, with indistinct, pale, eosinophilic cytoplasm, and ovoid nuclei with variably hyperchromatic chromatin and generally regular nuclear contours (figure 4C). The degree of nuclear atypia and hyperchromasia of these spindle cells varies between tumours, with most tumours showing only focal and/or mild atypia. However, prominent and/or diffuse atypia can be seen in approximately one-third of cases. ${ }^{35}$

The adipocytic component predominantly comprises mature-appearing adipocytes, but variation in adipocyte size can be seen (figure 4D).

Univacuolated or multivacuolated lipoblasts are present in almost half of cases, but usually in small numbers (figure 5A). Lipoblasts range from small, univacuolated or bivacuolated to larger, multivacuolated cells, with hyperchromatic scalloped 


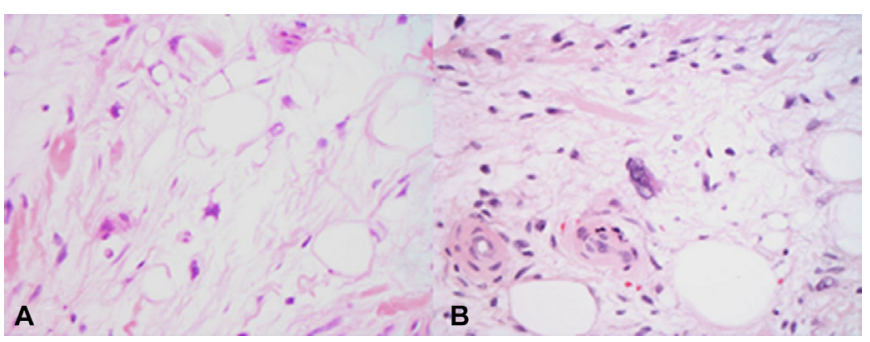

Figure 5 Univacuolated and multivacuolated lipoblastic cells are present in atypical spindle cell lipomatous tumour (A). Also bizarre, hyperchromatic, often multinucleated cells are encountered in either the adipocytic or spindle cell components (B).

nuclei and sharply punched-out vacuoles. In addition, in the majority of cases, bizarre, hyperchromatic, often multinucleated cells are scattered throughout the tumour, within the adipocytic or spindle cell components (figure $5 \mathrm{~B}$ ). ${ }^{35}$

Extracellular matrix is abundant in most tumours, varying widely from purely myxoid to predominantly collagenous. The amount of collagen present varies. The presence of bundles of ropey collagen is a rare finding. ${ }^{35}$

Thus, due to the extremely variable proportions of spindle cells, adipocytes, lipoblasts and extracellular matrix, as well as the variable quality of the extracellular matrix, there is a wide range of microscopic appearances of ASCLTs. These tumours can be paucicellular, with few, cytologically bland, spindle cells with minimal nuclear atypia, set in an abundant myxoid matrix with scattered mature adipocytes. They can bear a close resemblance to an SCL. At the other extreme, these tumours may be significantly more cellular, composed of numerous spindle cells showing diffuse, mild-to-moderate nuclear atypia, with variable presence of lipoblasts and less extracellular matrix. It is therefore extremely important to sample these fatty lesions with spindle cells thoroughly, if not in total.

At present, there are no reproducible morphological, immunohistochemical or molecular criteria to accurately subclassify atypical spindle cell lipomatous neoplasms into clinically meaningful subsets.

\section{Immunohistochemistry and cytogenetics}

The neoplastic cells express CD34 (65\%), S100 (40\%) and desmin (20\%). Loss of nuclear pRb expression is encountered in around $50 \%-60 \%$ of cases, correlating with $R B 1$ heterozygous deletion. However, it is important to note that either retention or loss of nuclear pRb staining is still compatible with the diagnosis of ASCLT. Weak and/or focal expression of MDM2 (5\%) or CDK4 $(5 \%)$ can be seen, but always in the absence of corresponding gene amplification. ${ }^{35}$ The combination of MDM 2 and CDK4 positivity together is not encountered in ASCLT. Typically, no nuclear p16 expression is seen in the adipocytes or the spindle-shaped tumour cells of ASCLT. ${ }^{8}$

Alterations such as $13 \mathrm{q}$ deletion with loss of $\mathrm{pRb}$, and monosomy 7 have been shown to be recurrent findings in these lesions. ${ }^{8} 3738$ FISH analysis does not demonstrate MDM2 or CD4K amplification. ${ }^{35}$ This latter finding separates ASCLT from liposarcoma.

\section{Differential diagnosis}

The morphological differential diagnosis of ASCLT is broad, and includes benign and malignant lesions (including low-grade malignant peripheral nerve sheath tumour, dermatofibrosarcoma protuberans, ALT/WDL, low-grade dedifferentiated liposarcoma (DDL))..$^{35}$

1. Spindle cell/pleomorphic lipomas as noted above can have slight atypia of the spindle cell component or bizarre cells in the pleomorphic variant. Both location and the loss of $\mathrm{pRb}$ are strongly in favour of the diagnosis of SC/PL (bearing in mind that approximately half of ASCLTs can also show loss of $\mathrm{pRb}$ ). Given that MDM2 is also not amplified in ASCLT, separation from SCL on morphological, immunohistochemical and cytogenetic grounds can be extremely difficult. If the lesion is cytologically bland and located in the neck/upper back region, then SCL is most likely. If located in the limbs and one is considering a diagnosis of SCL, then caution should be exercised, multiple sections examined for atypia and a diagnosis of ASCLT should be rendered.

2. Diffuse neurofibromas are often located within the dermis and subcutis of young patients, and are composed of a monomorphic proliferation of S100-positive spindle cells, with wavy/buckled nuclei. Adipocytic differentiation is rare, but entrapment of adjacent structures, including fat, may occur thus simulating a spindle cell and fat lesion. The detection of hyperplastic nerve bundles and Meissnerian corpuscles can aid in correctly identifying diffuse neurofibromas. ${ }^{35}$

3. Fat-forming SFT generally occurs in deep soft tissue and has a variably prominent adipocytic component. ${ }^{39}$ It has a 'patternless' architecture, characteristically variable cellularity and branching blood vessels, and is composed of bland spindle cells embedded in a collagenised stroma. Virtually, all SFTs demonstrate nuclear expression of STAT6, CD34, CD99 and bcl-2 by immunohistochemistry. ${ }^{26}$

4. Low-grade malignant peripheral nerve sheath tumours (MPNSTs) are usually better circumscribed and more cellular than ASCLTs. They are composed of slender spindle cells, with tapering/wavy nuclei exhibiting mild nuclear atypia, growing in fascicles and sheets, with characteristic perivascular accentuation. The lesional cells show variable S100 protein and SOX-10 positivity. ${ }^{40}$

5. Dermatofibrosarcoma protuberans (DFSP) arising in skin may show marked infiltration of underlying subcutaneous adipose tissue, mimicking an adipocytic neoplasm with a spindle cell component. The spindle cells of DFSP are typically monotonous, growing in a characteristic storiform pattern. The adipose tissue infiltrated by DFSP is benign and therefore, shows no cytological atypia and lacks variation in adipocyte size and shape. ${ }^{35}$ Up to $85 \%$ of DFSPs harbour the $\mathrm{t}(\mathrm{q} 21 ; \mathrm{q} 13)^{17} 22$ translocation.

6. Low-grade DDLs: a subset of DDLs morphologically may be indistinguishable from ASCLT on the cellular end of the spectrum. ${ }^{41}$ Morphological features favouring DDL are the presence of an abrupt transition from a well-differentiated adipocytic component into non-lipogenic spindle cell areas, and/or the presence of higher-grade areas and widespread cytological atypia. In contrast, the presence of lipogenesis intimately admixed with, rather than sharply demarcated from, the spindle cell component is characteristic of ASCLTs. DDL can be ruled out by demonstrating absence of MDM2 and CDK4 expression by immunohistochemistry, and lack of MDM2 amplification by FISH testing. This is a critical distinction, given the metastatic potential of DDLs.

\section{Treatment and prognosis}

ASCLT is a clinically low-grade lesion, which requires adequate surgical excision with clear margins due to its infiltrative growth 
pattern that can result in a low, but non-negligible, tendency for local recurrence, with reported rates of about $12 \%$ for incompletely removed lesions. ${ }^{3536}$ The risk for dedifferentiation and/ or metastasis is minimal. ${ }^{35} 36$

\section{Liposarcoma}

Liposarcomas are now grouped into several categories, namely: ALT or WDL, DDL, myxoid/round cell liposarcoma and pleomorphic liposarcoma.

Several fatty tumours with spindle cells, such as SCL, MTMF, cellular angiofibroma, ASCLT and WDL, all show considerable morphological overlap, thus warranting an overview attempting to delineate these entities.

\section{Atypical lipomatous tumour/well-differentiated liposarcoma Nomenclature}

The term ALT is preferred in superficial soft tissues that are amenable to wide local excision. Where curative resection cannot be achieved, the term WDL is used for the same lesion. ${ }^{11}$

WHO classifies WDL into three main subtypes: adipocytic, sclerosing and inflammatory. Adipocytic or lipoma-like liposarcoma consists of mature adipocytes, exhibiting variation in cell size and focal nuclear atypia and hyperchromasia. ${ }^{42}$ The sclerosing subtype comprises scattered distinctive bizarre stromal cells, associated with rare multivacuolated lipoblasts set in a fibrillar collagenous background. The inflammatory subtype contains a polyphenotypic lymphoplasmacytic infiltrate, with a predominance of B lymphocytes. ${ }^{42-44}$

\section{Clinical features}

ALT/WDL is a locally aggressive mesenchymal neoplasm occurring in deep-seated locations, such as in the retroperitoneum and mediastinum.

\section{Microscopic findings}

ALT/WDL is composed, entirely or in part, of an adipocytic proliferation with significant variation in adipocyte size (figure 6A) and a variable number of atypical, enlarged, hyperchromatic ('inky' nuclei) stromal cells, present in fibrous septae (figure 6B). Lipoblasts may or may not be present in ALT/WDL, and this is reflected in the current WHO diagnostic criteria for ALT/WDL, where lipoblasts are considered a helpful clue, but not a prerequisite, for the diagnosis. ${ }^{7}$ Myxoid change is well recognised in ALT/WDL, and when extensive, such cases may be mistaken for a myxoid liposarcoma (figure 7A). Various combinations of these features may occur and there are no significant clinicopathological, molecular or prognostic differences. ${ }^{45}$

\section{Immunohistochemistry and cytogenetics}

ALT/WDLs are characterised by supernumerary ring chromosomes or giant marker chromosomes that demonstrate amplifications in the chromosomal region 12q13-15 (figure 7B) ${ }^{46}$; these amplifications constantly affect MDM2 (100\%), and frequently affect CDK4 (90\%) and HMGA2, genes. Thus, ALT/WDLs demonstrate MDM2 and CDK4 overexpression by immunohistochemistry. However, MDM2 staining tends to be weak and scattered in ALT/WDLs. In addition, MDM2 staining may be difficult to interpret, especially if macrophages are present, as they can stain positively. This can be a pitfall in lipomas with secondary degenerative changes, where inflammatory cells and macrophages are positive for MDM $2 .{ }^{23}$ CDK4 immunohistochemistry has a low sensitivity and approximately $10 \%$ of ALT/ WDLs do not possess amplification of CDK4. ${ }^{47}$

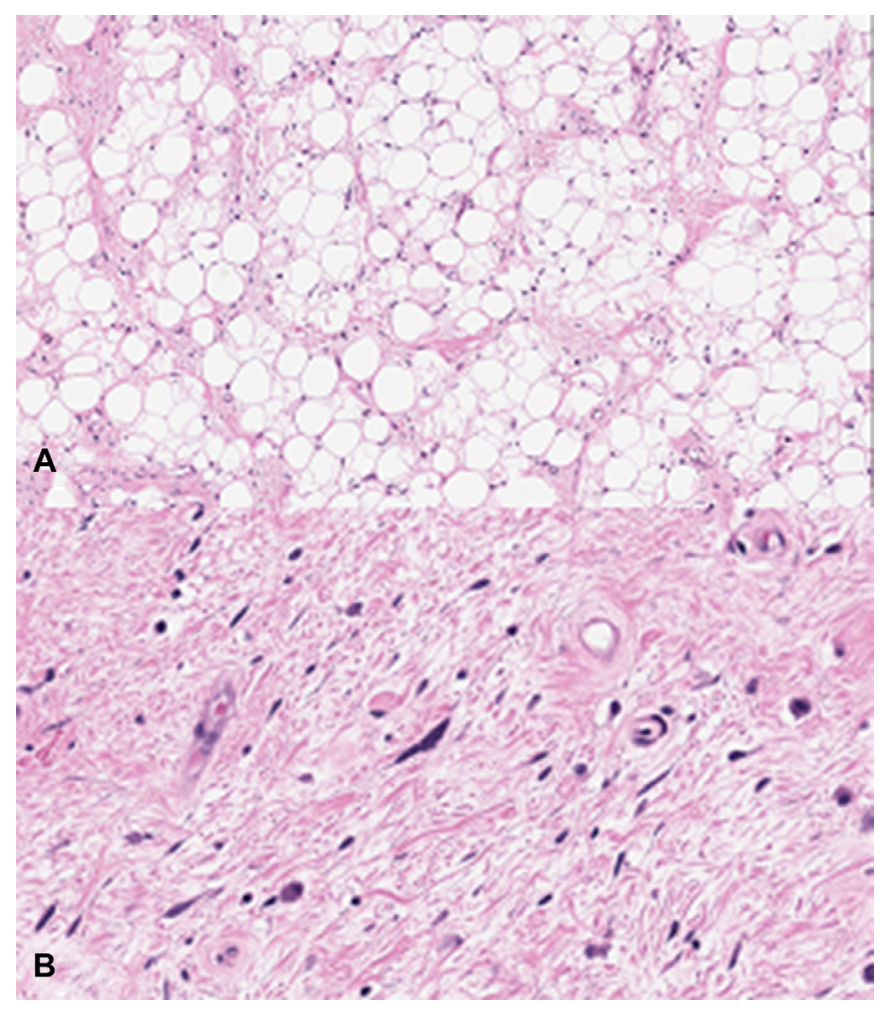

Figure 6 Atypical lipomatous tumour or well-differentiated liposarcoma contains adipocytes displaying significant variation in adipocyte size (A) with variable numbers of atypical, enlarged, hyperchromatic ('inky') nuclei stromal cells, often present in fibrous septae (B).

Amplification of MDM2 and CDK4 detection using FISH is the recommended gold standard methodology. ${ }^{48} 49$

$\mathrm{p} 16^{\mathrm{Ink} 4 \mathrm{~A}}(\mathrm{p} 16)$ is a transcript of the cyclin-dependent kinase inhibitor 2A (CDKN2A) gene and it inhibits cell cycle progression by binding to CDK4. ${ }^{50}$ It has been shown to be overexpressed in ALT-WDL by both quantitative reverse-transcription PCR and immunohistochemistry (figure 7C), and consequently, p16 has been proposed as a diagnostic marker. ${ }^{51}$ Pathologists should be aware of, and disregard, cytoplasmic staining that may be observed in endothelial and inflammatory cells. ${ }^{52}$

Of note, nuclear $\mathrm{pRb}$ expression is typically intact in ALT/ WDL (figure 7D).

\section{Differential diagnosis}

From a practical and patient management point of view, the most important differential diagnosis for ALT/WDL is a benign fatty tumour. The distinction between ALT/WDLs and benign adipocytic tumours is crucial because of differences in prognosis and treatment. In ALT/WDL, the atypical stromal cells may be poorly represented or absent and lipoblasts may not be seen. In lipomas, fibrosis, necrosis, inflammatory cells and pseudolipoblasts may be present. MDM2 gene amplification by FISH is an essential adjunct to prevent both underdiagnosis and overdiagnosis of ALT/WDL.

Clay et al suggest that FISH should be performed in the following scenarios pertaining to problematic lipomatous tumours: in recurrent 'lipomas'; in cases with equivocal cytological atypia; in lesions of the retroperitoneum, pelvis or abdomen; in any deep-seated tumour of the extremity measuring $>10 \mathrm{~cm}$ in a patient over the age of 50 years and in special clinical situations as directed by treating clinicians. ${ }^{53}$ 


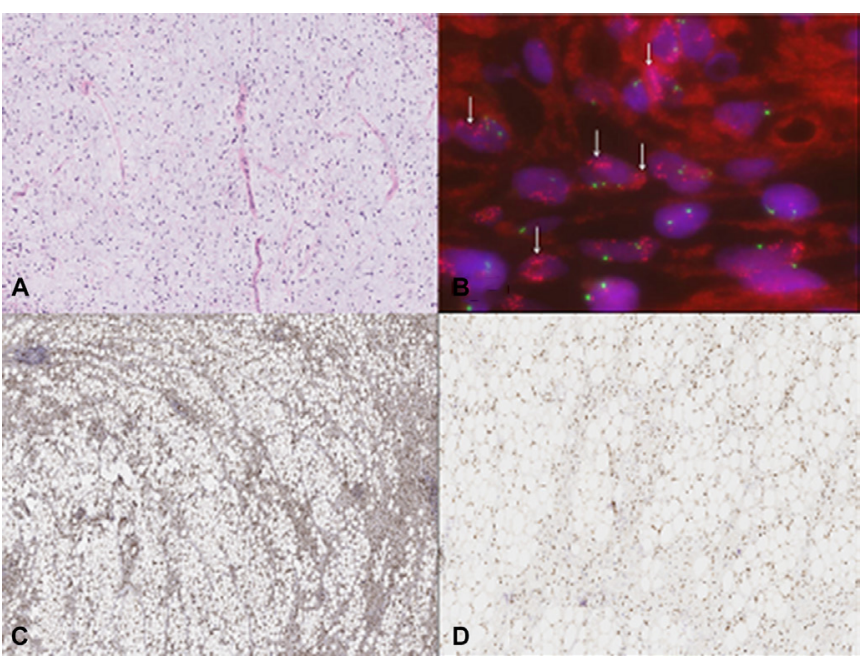

Figure 7 Myxoid stromal change is frequently encountered in atypical lipomatous tumour/well-differentiated liposarcoma, and can be extensive enough to resemble myxoid liposarcoma (A). Of diagnostic importance is the demonstration of MDM2 amplification by fluorescence in situ hybridisation (see arrows) (B). These lesions are p16 positive $(C)$ and show retention of nuclear retinoblastoma protein staining (D).

\section{Treatment and prognosis}

ALT/WDL has no metastatic potential and therefore such tumours are better classified as being of intermediate (but locally aggressive) biological potential. However, the overwhelming majority of retroperitoneal and mediastinal WDLs are ultimately associated with frequent local recurrence and significant mortality, even in the absence of dedifferentiation.

Dedifferentiation occurs in up to $10 \%$ of ALT/WDLs, with the risk of dedifferentiation being much higher in the retroperitoneum than elsewhere, almost certainly as a consequence of the longer lesional duration and larger tumour size at this site. ${ }^{11} 54$

\section{Dedifferentiated liposarcoma \\ Clinical features}

ALT/WDL and DDL form the largest subgroup of liposarcomas, and represent a morphological and behavioural spectrum representing one disease entity. ${ }^{55} 56$ The classic definition of DDL states that it is a 'non-lipogenic sarcoma' arising in association with a WDL/an ALT.

DDLs present most frequently in middle-aged and older adults, with an equal gender distribution. They are typically large $(>10 \mathrm{~cm})$, multinodular masses. ${ }^{54}$ The retroperitoneum is considered the most common site of DDLs (where a spindle cell sarcoma is a DDL until proven otherwise), but they also occur in other locations, including the extremities, the paratesticular region, and, more rarely, the trunk (including mediastinum and thorax), head and neck. ${ }^{54}$ 57-59 DDLs typically surround adjacent visceral structures but frank invasion of these structures is rare. 54

Up to $90 \%$ of DDLs present as de novo neoplasms while the remainder occur as a recurrence of a pre-existing ALT/WDL, after an average interval of 7.7 years. ${ }^{41}$ Dedifferentiation can occur in up to $10 \%$ of ALT/WDLs at any site, ${ }^{54}$ with the risk being greater in tumours that are deep-seated, particularly those in the retroperitoneum, where this risk is approximately $28 \% .^{545960}$

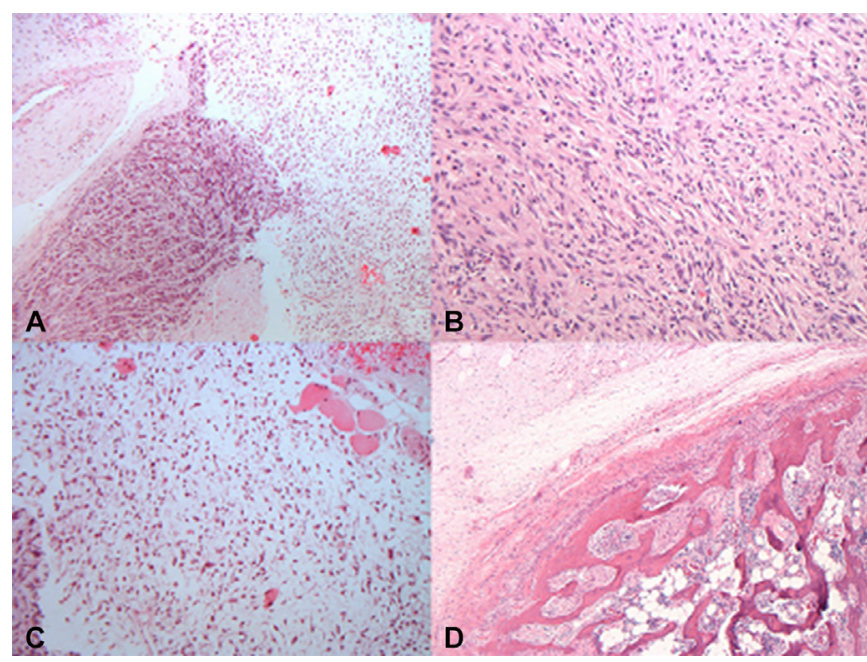

Figure 8 Dedifferentiated liposarcoma (DDL) is characterised by a sharp demarcation between the well-differentiated and dedifferentiated components (A). The dedifferentiated component is made up of spindle cells with mild-to-moderate atypia in fascicles resembling a spindle sarcoma (B), set within a myxoid stroma (C). Unusual histological accompaniments include metaplastic bone (D).

\section{Microscopic findings}

DDLs show a spectrum of morphological appearances, although the majority show features of undifferentiated pleomorphic sarcoma or spindle cell sarcoma not otherwise specified, ${ }^{54}$ with high-to-moderate cellularity, and with cells disposed in loose fascicles, patternless distributions or sometimes with a storiform architecture, within variably fibrous stroma. This is sharply demarcated from the well-differentiated component (figure 8A). Prominent cellularity with nuclear pleomorphism andmoderate to marked cytological atypia are seen, the mitotic index is variable, and necrosis can also be seen (figure 8B). ${ }^{61} 62$

Prominent myxoid stroma can be a feature of DDLs (figure $8 \mathrm{C}$ ). When present, atypical spindle or ovoid cells with a myxofibrosarcoma-like morphology are present within the myxoid stroma. In addition, a prominent coarse plexiform vascular pattern (similar to that seen in myxofibrosarcoma), and sometimes a 'pulmonary oedema-like' pattern (similar to that of myxoid liposarcoma) may be seen. ${ }^{63}$

Metaplastic bone, meningothelial-like whorls and metaplastic cartilage may be seen rarely (figure $8 \mathrm{D}){ }^{64-66}$

DDLs may exhibit heterologous differentiation towards other mesenchymal lineages, including chondroid, osteoid, myoid (both smooth and skeletal muscle differentiation). ${ }^{416768}$ Rarely, angiosarcomatous differentiation has been described. ${ }^{69}$

A minority of DDLs are of low cellularity and comprise only histologically 'low-grade' areas resembling fibromatosis or low-grade fibromyxoid sarcoma. ${ }^{41} 70$ Low cellularity DDLs are composed of sparsely to moderately cellular proliferations comprising loose fascicles or patternless distributions of fibroblast-like spindle cells with mild nuclear atypia and low mitotic activity. $^{54}$

A small subset of cases may demonstrate lipoblastic differentiation within the dedifferentiated component (ie, rare cases may be 'lipogenic'). ${ }^{72}$ This finding is referred to as DDL with 'homologous lipoblastic differentiation' or 'pleomorphic liposarcoma-like features'. ${ }^{67} 73$ 


\section{Immunohistochemistry and cytogenetics}

DDLs can show variable expression of CD34, with focal positivity for smooth muscle actin and desmin, while S100 protein is absent in non-lipogenic areas of DDLs. ${ }^{54}$ Most DDLs (>90\%) show immunohistochemical expression of MDM2 and CDK4 and there is strong correlation between expression of these immunohistochemical stains and their gene amplification status. $^{2374}$ The combination of p16 with CDK4 and MDM2 is useful in distinguishing DDLs from other adipocytic neoplasms, and is more sensitive than the use of CDK4 and MDM2 alone. Approximately 93\% of DDLs express at least two antigens of this triad of markers, and p16 has been shown to be the most sensitive and specific marker for detecting DDLs. ${ }^{74}$

The assessment of MDM2 gene amplification by FISH is a highly useful adjunctive diagnostic tool for the diagnosis of DDL. ${ }^{75}$

A recent paper details the results of whole-exome sequencing performed on 206 sarcomas, including 50 DDLs, all of which were defined by $12 \mathrm{q} 13-15$ amplifications, including highly recurrent copy-number gains or amplification of MDM2 (100\% of samples), CDK4 (92\%) and HMGA2 (76\%), as well as FRS2 (96\%) and NAV3 (60\%). ${ }^{1}$ Other frequent somatic copy-number alterations involved genes reported to inhibit adipocyte differentiation, namely PTPRQ (46\%), JUN (42\%), DDIT3 (32\%), CEBPA (24\%) and YAP1 (16\%). Recurrent deletions of ATRX (30\%), NF1 (28\%) and CDKN2A (44\%) were also detected. ${ }^{1}$

\section{Differential diagnosis}

The diverse histological patterns of DDL can lead to difficulty in distinguishing it from other soft tissue sarcomas, particularly in patients without an antecedent history of ALT/WDL, and in tumours in which a component of ALT/WDL is lacking. ${ }^{54}$

Morphological clues to identify DDLs are the presence of an abrupt, sharp transition from a well-differentiated adipocytic component into non-lipogenic spindle cell areas, and/ or the presence of higher-grade areas, widespread atypia and varied morphology or cellular pleomorphism. ${ }^{54}$ Essentially all neoplasms in the differential diagnosis of DDL will lack any adjacent WDL, and will tend to lack expression of p16, CDK4 and MDM2 by immunohistochemistry and lack evidence of MDM2 amplification by FISH. ${ }^{54}$

It is worth bearing in mind that it has been shown that most neoplasms arising in the retroperitoneum that are diagnosed as undifferentiated pleomorphic sarcoma (so-called 'malignant fibrous histiocytoma'), in fact represent DDLs. ${ }^{76}$

\section{Treatment and prognosis}

The diagnosis of DDL is prognostically significant, as it has a lower tendency towards local recurrence and metastasis, when compared with some other liposarcomas and with morphologically similar pleomorphic soft tissue sarcomas such as leiomyosarcomas or undifferentiated pleomorphic sarcomas. ${ }^{62}$

Radical surgical excision remains the mainstay of treatment, although radiotherapy is considered a valuable treatment option in retroperitoneal DDLs. ${ }^{54}$

DDL behaves in a more aggressive manner than ALT/WDL, with a greater propensity for local recurrence (in approximately $41 \%$ of cases) as well as the capacity to metastasize (in 15\%-30\% of cases), but it tends to behave less aggressively than other pleomorphic sarcomas. ${ }^{415462}$

Macroscopic tumour clearance at any site has been shown to be significantly associated with reduced local recurrence and improved survival. ${ }^{77} \mathrm{~A}$ recent study has shown that retroperitoneal liposarcomas with myogenic differentiation, and particularly those with a rhabdomyoblastic component, have a significantly poorer outcome. ${ }^{78}$ The most important adverse prognostic factor has been reported to be a location in the retroperitoneum, with tumours here showing significantly worse survival than those at other anatomic sites. ${ }^{41}$

Even histologically, low-grade DDLs have been shown to have the capacity to metastasize and to behave like traditional DDLs, rather than like WDLs. ${ }^{4179}$

Take home messages

- Fatty tumours with a spindle cell component encompass both benign and malignant tumours.

- Immunohistochemistry and/or cytogenetic analysis is critical for the correct diagnosis of spindle cell fatty tumours.

- Spindle cell lipoma/mammary-type myofibroblastoma/ cellular angiofibroma/atypical spindle cell lipomatous tumours (ASCLT) are lesions that are characterised by spindle cells (often bland) and fat, nuclear retinoblastoma protein loss and absence of amplification of MDM2 and CDK4.

- ASCLT is a newly created group of tumours incorporating spindle cell liposarcoma.

- ASCLT can mimic spindle cell lipoma morphologically, immunohistochemically and cytogenetically. Such cases are separated by location of the lesion.

- Thorough sampling of fatty lesions with spindle cells is advocated in view of the variable and overlapping features.

\section{Handling editor Cheok Soon Lee.}

Contributors AJM and RC contributed equally to the conceptualising and writing of the manuscript.

Funding This research received no specific grant from any funding agency in the public, commercial or not-for-profit sectors.

Competing interests None declared.

Provenance and peer review Commissioned; internally peer reviewed. Data sharing statement None.

(c) Article author(s) (or their employer(s) unless otherwise stated in the text of the article) 2018. All rights reserved. No commercial use is permitted unless otherwise expressly granted.

\section{REFERENCES}

1 Abeshouse A, Adebamowo C, Adebamowo SN, et al. Cancer genome atlas research network. Comprehensive and integrated genomic characterization of adult soft tissue sarcomas. Cell 2017;171:950-65.

2 Clark MA, Fisher C, Judson I, et al. Soft-tissue sarcomas in adults. N Engl J Med 2005;353:701-11.

3 Mandahl N, Mertens F, Willén H, et al. A new cytogenetic subgroup in lipomas: loss of chromosome 16 material in spindle cell and pleomorphic lipomas. J Cancer Res Clin Oncol 1994;120:707-11.

4 Fletcher CD, Martin-Bates E. Spindle cell lipoma: a clinicopathological study with some original observations. Histopathology 1987;11:803-17.

5 Yang H, Yu L. Cutaneous and Superficial Soft Tissue CD34+ Spindle Cell Proliferation. Arch Pathol Lab Med 2017;141:1092-100.

6 French CA, Mentzel T, Kutzner H, et al. Intradermal spindle cell/pleomorphic lipoma: a distinct subset. Am J Dermatopathol 2000;22:496-502.

7 Michal M, Kazakov DV, Hadravsky L, et al. Lipoblasts in spindle cell and pleomorphic lipomas: a close scrutiny. Hum Pathol 2017;65:140-6.

8 Creytens D, van Gorp J, Savola S, et al. Atypical spindle cell lipoma: a clinicopathologic, immunohistochemical, and molecular study emphasizing its relationship to classical spindle cell lipoma. Virchows Arch 2014;465:97-108.

9 Adsay NV, Hasteh F, Cheng JD, et al. Lymphoepithelial cysts of the pancreas: a report of 12 cases and a review of the literature. Mod Pathol 2002;15:492-501.

10 Howitt BE, Fletcher CD. Mammary-type Myofibroblastoma: Clinicopathologic Characterization in a Series of 143 Cases. Am J Surg Pathol 2016:40:361-7.

11 Fletcher CD. The evolving classification of soft tissue tumours: an update based on the new WHO classification. Histopathology 2006;48:3-12. 
12 Magro G, Foschini MP, Eusebi V. Palisaded myofibroblastoma of the breast: a tumor closely mimicking schwannoma: Report of 2 cases. Hum Pathol 2013;44:1941-6.

13 Magro G. Epithelioid-cell myofibroblastoma of the breast: expanding the morphologic spectrum. Am J Surg Pathol 2009;33:1085-92.

14 Magro G. Mammary myofibroblastoma: a tumor with a wide morphologic spectrum. Arch Pathol Lab Med 2008;132:1813-20.

15 McMenamin ME, DeSchryver K, Fletcher CD. Fibrous Lesions of the Breast: A Review. Int J Surg Pathol 2000;8:99-108.

16 Chen BJ, Mariño-Enríquez A, Fletcher CD, et al. Loss of retinoblastoma protein expression in spindle cell/pleomorphic lipomas and cytogenetically related tumors: an immunohistochemical study with diagnostic implications. Am J Surg Pathol 2012;36:1119-28.

17 McMenamin ME, Fletcher CD. Mammary-type myofibroblastoma of soft tissue: a tumor closely related to spindle cell lipoma. Am J Surg Pathol 2001;25:1022-9.

18 Flucke U, van Krieken JH, Mentzel T. Cellular angiofibroma: analysis of 25 cases emphasizing its relationship to spindle cell lipoma and mammary-type myofibroblastoma. Mod Pathol 2011;24:82-9.

19 Maggiani F, Debiec-Rychter M, Vanbockrijck M, et al. Cellular angiofibroma: another mesenchymal tumour with $13 q 14$ involvement, suggesting a link with spindle cell lipoma and (extra)-mammary myofibroblastoma. Histopathology 2007:51:410-2.

20 Iwasa Y, Fletcher CD. Cellular angiofibroma: clinicopathologic and immunohistochemical analysis of 51 cases. Am J Surg Pathol 2004;28:1426-35.

21 Brennan MF, Antonescu CR, Moraco N, et al. Lessons learned from the study of 10,000 patients with soft tissue sarcoma. Ann Surg 2014;260:416-22.

22 Huang K, Wang CM, Chen JG, et al. Prognostic factors influencing event-free survival and treatments in desmoid-type fibromatosis: analysis from a large institution. Am J Surg 2014;207:847-54.

23 Binh MB, Sastre-Garau X, Guillou L, et al. MDM2 and CDK4 immunostainings are useful adjuncts in diagnosing well-differentiated and dedifferentiated liposarcoma subtypes: a comparative analysis of 559 soft tissue neoplasms with genetic data. Am J Surg Pathol 2005;29:1340-7.

24 Amary MF, Pauwels P, Meulemans E, et al. Detection of beta-catenin mutations in paraffin-embedded sporadic desmoid-type fibromatosis by mutation-specific restriction enzyme digestion (MSRED): an ancillary diagnostic tool. Am J Surg Pathol 2007;31:1299-309.

25 Fritchie KJ, Carver P, Sun Y, et al. Solitary fibrous tumor: is there a molecular relationship with cellular angiofibroma, spindle cell lipoma, and mammary-type myofibroblastoma? Am J Clin Pathol 2012;137:963-70.

26 Doyle LA, Vivero M, Fletcher CD, et al. Nuclear expression of STAT6 distinguishes solitary fibrous tumor from histologic mimics. Mod Pathol 2014;27:390-5.

27 Robinson DR, Wu YM, Kalyana-Sundaram S, et al. Identification of recurrent NAB2STAT6 gene fusions in solitary fibrous tumor by integrative sequencing. Nat Genet 2013:45:180-5.

28 Eversole LR. Cellular angiofibroma of oral mucosa: report of two cases. Head Neck Pathol 2009:3:136-9.

29 Kandil DH, Kida M, Laub DR, et al. Sarcomatous transformation in a cellular angiofibroma: a case report. J Clin Pathol 2009;62:945-7.

30 Val-Bernal JF, Rubio S, Garijo MF, et al. Extragenital subcutaneous cellular angiofibroma. Case report. APMIS 2007;115:254-8.

31 Fletcher CD, Tsang WY, Fisher C, et al. Angiomyofibroblastoma of the vulva. A benign neoplasm distinct from aggressive angiomyxoma. Am J Surg Pathol 1992;16:373-82.

32 Nucci MR, Fletcher CD. Vulvovaginal soft tissue tumours: update and review. Histopathology 2000;36:97-108.

33 Chen E, Fletcher CD. Cellular angiofibroma with atypia or sarcomatous transformation: clinicopathologic analysis of 13 cases. Am J Surg Pathol 2010;34:1-14.

34 McCluggage WG, Ganesan R, Hirschowitz L, Rollason TP, et al. Cellular angiofibroma and related fibromatous lesions of the vulva: report of a series of cases with a morphological spectrum wider than previously described. Histopathology 2004:45:360-8.

35 Mariño-Enriquez A, Nascimento AF, Ligon AH, et al. Atypical Spindle Cell Lipomatous Tumor: Clinicopathologic Characterization of 232 Cases Demonstrating a Morphologic Spectrum. Am J Surg Pathol 2017;41:234-44.

36 Dei Tos AP, Mentzel T, Newman PL, et al. a hitherto unrecognized variant of liposarcoma. Analysis of six cases. Am J Surg Pathol 1994;18:913-21.

37 Deyrup AT, Chibon F, Guillou L, et al. Fibrosarcoma-like lipomatous neoplasm: a reappraisal of so-called spindle cell liposarcoma defining a unique lipomatous tumor unrelated to other liposarcomas. Am J Surg Pathol 2013;37:1373-8.

38 Mentzel T, Palmedo G, Kuhnen C. Well-differentiated spindle cell liposarcoma ('atypical spindle cell lipomatous tumor') does not belong to the spectrum of atypical lipomatous tumor but has a close relationship to spindle cell lipoma: clinicopathologic, immunohistochemical, and molecular analysis of six cases. Mod Pathol 2010;23:729-36.

39 Guillou L, Gebhard S, Coindre JM. Lipomatous hemangiopericytoma: a fat-containing variant of solitary fibrous tumor? Clinicopathologic, immunohistochemical, and ultrastructural analysis of a series in favor of a unifying concept. Hum Pathol 2000;31:1108-15
40 Ducatman BS, Scheithauer BW, Piepgras DG, et al. Malignant peripheral nerve sheath tumors. A clinicopathologic study of 120 cases. Cancer 1986;57:2006-21.

41 Henricks WH, Chu YC, Goldblum JR, et al. Dedifferentiated liposarcoma: a clinicopathological analysis of 155 cases with a proposal for an expanded definition of dedifferentiation. Am J Surg Pathol 1997;21:271-81.

42 Dei Tos AP, Doglioni C, Piccinin S, et al. Coordinated expression and amplification of the MDM2, CDK4, and HMGI-C genes in atypical lipomatous tumours. J Pathol 2000;190:531-6.

43 Kraus MD, Guillou L, Fletcher CD. Well-differentiated inflammatory liposarcoma: an uncommon and easily overlooked variant of a common sarcoma. Am J Surg Pathol 1997;21:518-27.

44 Argani P, Facchetti F, Inghirami G, et al. Lymphocyte-rich well-differentiated liposarcoma: report of nine cases. Am J Surg Pathol 1997:21:884-95.

45 Rosai J, Akerman M, Dal Cin P, et al. Combined morphologic and karyotypic study of 59 atypical lipomatous tumors. Evaluation of their relationship and differential diagnosis with other adipose tissue tumors (a report of the CHAMP Study Group). Am J Surg Pathol 1996;20:1182-9.

46 Mertens F, Panagopoulos I, Mandahl N. Genomic characteristics of soft tissue sarcomas. Virchows Arch 2010;456:129-39.

47 Aleixo PB, Hartmann AA, Menezes IC, et al. Can MDM2 and CDK4 make the diagnosis of well differentiated/dedifferentiated liposarcoma? An immunohistochemical study on 129 soft tissue tumours. J Clin Pathol 2009:62:1127-35.

48 Sirvent N, Coindre JM, Maire G, et al. Detection of MDM2-CDK4 amplification by fluorescence in situ hybridization in 200 paraffin-embedded tumor samples: utility in diagnosing adipocytic lesions and comparison with immunohistochemistry and realtime PCR. Am J Surg Pathol 2007:31:1476-89.

49 Weaver J, Rao P, Goldblum JR, et al. Can MDM2 analytical tests performed on core needle biopsy be relied upon to diagnose well-differentiated liposarcoma? Mod Pathol 2010;23:1301-6

50 Kim WY, Sharpless NE. The regulation of INK4/ARF in cancer and aging. Cell 2006;127:265-75.

51 Louis-Brennetot C, Coindre JM, Ferreira C, et al. The CDKN2A/CDKN2B/ CDK4/CCND1 pathway is pivotal in well-differentiated and dedifferentiated liposarcoma oncogenesis: an analysis of 104 tumors. Genes Chromosomes Cancer 2011:50:896-907.

52 He M, Aisner S, Benevenia J, et al. p16 immunohistochemistry as an alternative marker to distinguish atypical lipomatous tumor from deep-seated lipoma. Appl Immunohistochem Mol Morphol 2009;17:51-6.

53 Clay MR, Martinez AP, Weiss SW, et al. MDM2 Amplification in Problematic Lipomatous Tumors: Analysis of FISH Testing Criteria. Am J Surg Pathol 2015:39:1433-9.

54 Thway K, Jones RL, Noujaim J, et al. Dedifferentiated Liposarcoma: Updates on Morphology, Genetics, and Therapeutic Strategies. Adv Anat Pathol 2016;23:30-40.

55 Nascimento AG, liposarcoma D Semin Diagn Pathol 2001;18:263-6.

56 Laurino L, Furlanetto A, Orvieto E, et al. Well-differentiated liposarcoma (atypical lipomatous tumors). Semin Diagn Pathol 2001;18:258-62.

57 Boland JM, Colby TV, Folpe AL. Liposarcomas of the mediastinum and thorax: a clinicopathologic and molecular cytogenetic study of 24 cases, emphasizing unusual and diverse histologic features. Am J Surg Pathol 2012;36:1395-403.

58 Montgomery E, Fisher C. Paratesticular liposarcoma: a clinicopathologic study. Am J Surg Pathol 2003;27:40-7

59 Weiss SW, Rao VK. Well-differentiated liposarcoma (atypical lipoma) of deep soft tissue of the extremities, retroperitoneum, and miscellaneous sites. A follow-up study of 92 cases with analysis of the incidence of "dedifferentiation". Am J Surg Pathol 1992:16:1051-8.

60 Lucas DR, Nascimento AG, Sanjay BK, et al. The Mayo Clinic experience with 58 cases. Am J Clin Pathol 1994;102:677-83.

61 Hasegawa T, Seki K, Hasegawa F, et al. Dedifferentiated liposarcoma of retroperitoneum and mesentery: varied growth patterns and histological grades-a clinicopathologic study of 32 cases. Hum Pathol 2000;31:717-27.

62 McCormick D, Mentzel T, Beham A, et al. Dedifferentiated liposarcoma. Clinicopathologic analysis of 32 cases suggesting a better prognostic subgroup among pleomorphic sarcomas. Am J Surg Pathol 1994;18:1213-23.

63 Sioletic S, Dal Cin P, Fletcher CD, et al. Well-differentiated and dedifferentiated liposarcomas with prominent myxoid stroma: analysis of 56 cases. Histopathology 2013:62:287-93.

64 Nascimento AG, Kurtin PJ, Guillou L, et al. Dedifferentiated liposarcoma: a report of nine cases with a peculiar neurallike whorling pattern associated with metaplastic bone formation. Am J Surg Pathol 1998;22:945-55.

65 Fanburg-Smith JC, Miettinen M. Liposarcoma with meningothelial-like whorls: a study of 17 cases of a distinctive histological pattern associated with dedifferentiated liposarcoma. Histopathology 1998;33:414-24.

66 Thway K, Robertson D, Thway Y, et al. Dedifferentiated liposarcoma with meningothelial-like whorls, metaplastic bone formation, and CDK4, MDM2, and p16 expression: a morphologic and immunohistochemical study. Am J Surg Pathol 2011;35:356-63.

67 Mariño-Enríquez A, Fletcher CD, Dal Cin P, et al. Dedifferentiated liposarcoma with "homologous" lipoblastic (pleomorphic liposarcoma-like) differentiation: 
clinicopathologic and molecular analysis of a series suggesting revised diagnostic criteria. Am I Surg Pathol 2010;34:1122-31.

68 Binh MB, Guillou L, Hostein I, et al. Dedifferentiated liposarcomas with divergent myosarcomatous differentiation developed in the internal trunk: a study of 27 cases and comparison to conventional dedifferentiated liposarcomas and leiomyosarcomas. Am I Surg Pathol 2007;31:1557-66.

69 Evans HL, Khurana KK, Kemp BL, et al. Heterologous elements in the dedifferentiated component of dedifferentiated liposarcoma. Am J Surg Pathol 1994;18:1150-7.

70 Fukunaga M. Histologically low-grade dedifferentiated liposarcoma of the retroperitoneum. Pathol Int 2001;51:392-5.

71 Azumi N, Curtis J, Kempson RL, et al. Atypical and malignant neoplasms showing lipomatous differentiation. A study of 111 cases. Am I Surg Pathol 1987;11:161-2.

72 Doyle LA. Sarcoma classification: an update based on the 2013 World Health Organization Classification of Tumors of Soft Tissue and Bone. Cancer 2014;120:1763-74.

73 Boland JM, Weiss SW, Oliveira AM, et al. Liposarcomas with mixed well-differentiated and pleomorphic features: a clinicopathologic study of 12 cases. Am J Surg Pathol 2010:34:837-43.
74 Thway K, Flora R, Shah C, et al. Diagnostic utility of p16, CDK4, and MDM2 as an immunohistochemical panel in distinguishing well-differentiated and dedifferentiated liposarcomas from other adipocytic tumors. Am J Surg Pathol 2012:36:462-9.

75 Weaver J, Downs-Kelly E, Goldblum JR, et al. Fluorescence in situ hybridization for MDM2 gene amplification as a diagnostic tool in lipomatous neoplasms. Mod Pathol 2008;21:943-9.

76 Coindre JM, Mariani O, Chibon F, et al. Most malignant fibrous histiocytomas developed in the retroperitoneum are dedifferentiated liposarcomas: a review of 25 cases initially diagnosed as malignant fibrous histiocytoma. Mod Pathol 2003;16:256-62.

77 Neuhaus SJ, Barry P, Clark MA, et al. Surgical management of primary and recurrent retroperitoneal liposarcoma. Br J Surg 2005;92:246-52.

78 Gronchi A, Lo Vullo S, Fiore M, et al. Aggressive surgical policies in a retrospectively reviewed single-institution case series of retroperitoneal soft tissue sarcoma patients. J Clin Oncol 2009;27:24-30.

79 Elgar F, Goldblum JR. Well-differentiated liposarcoma of the retroperitoneum: a clinicopathologic analysis of 20 cases, with particular attention to the extent of lowgrade dedifferentiation. Mod Pathol 1997;10:113-20. 